\title{
Comprehensive analysis of whole genome methylation in mouse blastocysts cultured with four different constituents following in vitro fertilization
}

Yu Horibe ${ }^{1,2^{*}}$, Kazuhiko Nakabayashi ${ }^{2}$, Miyuki Arai ${ }^{2}$, Kohji Okamura $^{2}$, Kazunori Hashimoto ${ }^{1}$, Hideo Matsui ${ }^{1}$ and Kenichiro Hata ${ }^{2}$

\begin{abstract}
Background: With the development of assisted reproductive technology (ART), diseases believed to be caused by ART have begun to be identified as imprinted disease. However, no conclusion has been reached. So we sought to determine whether ART procedures disturb gene methylation and whether imprinted genes alone are selectively disturbed. To examine whether the constituents of the culture medium contribute to the changes in methylation, we used a mouse model to conduct IVF and comprehensively analyzed 5'-C-phosphate-G-3' (CpG) by reduced representation bisulfite sequencing (RRBS) using a second-generation sequencer to determine changes in methylation using four types of culture media with different amino acid constituents.

Results: We cultured ova to the blastocyst stage in a mouse model in culture media with four different amino acid constituents. Each culture medium included (1) KSOM culture medium (NoAA), (2) KSOM media + essential amino acids (EAAs), (3) KSOM medium + non-essential amino acids (NEAAs), or (4) KSOM medium + EAAs + NEAAs (AllAA) analyzed by reduced representation bisulfite sequencing. The results showed that (1) there were many regions that maintained hypermethylation with NEAAs, (2) there was little effect of demethylation on reprogramming in the 5' UTR and promoter regions, and (3) specific changes were observed in imprinted genes such as Nnat and Nespas.

Conclusions: Compared with EAAs, NEAAs could protect genes from demethylation caused by reprogramming. On the imprinted genes, methylation of the promoter region of $\mathrm{H} 19$ was decreased by NEAAs, suggesting that specific genes were prone to changes in methylation. It was suggested that these changes could provide similar results in humans. Further studies are needed to understand how changes in methylation may affect gene expression profiles.
\end{abstract}

Keywords: Blastocyst, Culture media, Imprinted gene, IVF, Methylation

\section{Background}

In 1978, Robert Edwards and Patrick Steptoe, an obstetrician and a gynecologist, developed basic assisted reproductive technology (ART) [1]. Since then, various methods have been employed to improve fertility rate. These include changes in the timing of ovum collection, changes in the types and numbers of ovulation-inducing agents, in vitro fertilization (IVF) and intracytoplasmic

\footnotetext{
* Correspondence: doyouknowphy@gmail.com

${ }^{1}$ Tokyo Women's Medical University, 8-1 Kawada-cho, Shinjuku, Tokyo, Japan ${ }^{2}$ National Center for Child Health and Development, Tokyo, Japan
}

sperm injection (ICSI) as part of IVF, and types of culture media used. The number of births through ART till date exceeds 5 million and accounts for $3 \%$ of births in Japan. According to various comparative studies, fertility rate outcomes have improved. However, in recent years, diseases believed to be caused by ART have begun to be identified [2]. While there are various phenotypes, epigenetic disruption caused by a series of ART procedures is mainly believed to be the underlying cause of disease. In particular, it is believed that the disruption of imprinted gene expression causes diseases 
such as Beckwith-Wiedemann syndrome [3]. Researchers worldwide have reported a causal relationship with imprinted genes; however, the incidence of imprinting defect is extremely low [4]. No conclusion has been reached because it is difficult to identify a clear relationship between imprinting defects and methylation. However, we sought to determine whether ART procedures disturb gene methylation and whether imprinted genes alone are selectively disturbed. To examine whether the constituents of the culture medium contribute to the changes in methylation, we used a mouse model to conduct IVF and comprehensively analyzed $5^{\prime}-\mathrm{C}$-phosphate-G-3' (CpG) by reduced representation bisulfite sequencing (RRBS) using a second-generation sequencer to determine changes in methylation using four types of culture media with different amino acid constituents.

\section{Methods}

\section{Ovulation induction and ovum collection}

Blastocysts were obtained by mating 8-week-old female and male mice from the Institute for Cancer Research (Charles River Laboratories Japan). Mice were exposed to daylight in the laboratory for $12 \mathrm{~h}(8 \mathrm{am}$ to $8 \mathrm{pm})$. The incubator temperature was set at $37^{\circ} \mathrm{C}$ with $\mathrm{CO}_{2}$ at $5 \%$. Ovarian stimulation was performed using pregnant mare's serum gonadotropin $7.5 \mathrm{IU}$ and human serum chorionic gonadotropin 7.5 IU. Pregnant mare's serum gonadotropin was injected in female mice; $48 \mathrm{~h}$ later, human serum chorionic gonadotropin was injected. Seventeen hours later, the cervical spines of the female mice were dislocated, after which oocyte masses were collected without delay from the ampulla of the fallopian tube. The ova were left for $1 \mathrm{~h}$ in human tubal fluid culture; next, IVF was performed using sperm insemination. The preparation was stored in an incubator for $2 \mathrm{~h}$ and $30 \mathrm{~min}$; the fragmented ova were removed and the fertilized eggs alone were selected. Eggs were divided into four groups, each flushed with a culture medium containing a different concentration of amino acids: (1) NoAAs $=$ KSOM medium (EmbryoMax ${ }^{\bullet}$ KSOM Embryo Culture (1X), Merck Millipore), (2) EAAs = KSOM medium + Essential Amino Acid (MEM Amino Acids Solution, life technologies), (3) NEAAs = KSOM medium + Non-Essential Amino Acid (MEM NonEssential Amino Acids Solution, life technologies), and (4) AllAAs = KSOM medium + Essential Amino Acid + Non-Essential Amino Acid (Table 1).

After flushing with these media, the preparation was cultured until the $\mathrm{E}=3.5$ blastocyst stage. After culturing, in accordance with the Gardner's grading system, fragmented ova and embryos that stopped growing were removed and mature blastocysts exhibiting good cleavage were selected [5].
Table 1 Constituent of essential and non-essential amino acid solution

Essential amino acid solution

https://www.thermofisher.com/jp/ja/home/technical-resources/mediaformulation.164.html

\begin{tabular}{|c|c|c|c|}
\hline Components & $\begin{array}{l}\text { Molecular } \\
\text { weight }\end{array}$ & $\begin{array}{l}\text { Concentration } \\
(\mathrm{mg} / \mathrm{L})\end{array}$ & $\mathrm{mM}$ \\
\hline L-Arginine hydrochloride & 211.0 & 6320.0 & 29.952606 \\
\hline L-Cystine & 240.0 & 1200.0 & 5.0 \\
\hline $\begin{array}{l}\text { L-Histidine } \\
\text { hydrochloride- } \mathrm{H}_{2} \mathrm{O}\end{array}$ & 210.0 & 2100.0 & 10.0 \\
\hline L-Isoleucine & 131.0 & 2620.0 & 20.0 \\
\hline L-Leucine & 131.0 & 2620.0 & 20.0 \\
\hline L-Lysine hydrochloride & 183.0 & 3625.0 & 19.808743 \\
\hline L-Methionine & 149.0 & 755.0 & 5.067114 \\
\hline L-Phenylalanine & 165.0 & 1650.0 & 10.0 \\
\hline L-Threonine & 119.0 & 2380.0 & 20.0 \\
\hline L-Tryptophan & 204.0 & 510.0 & 2.5 \\
\hline L-Tyrosine & 181.0 & 1800.0 & 9.944752 \\
\hline L-Valine & 117.0 & 2340.0 & 20.0 \\
\hline
\end{tabular}

Non-essential amino acid solution

https://www.thermofisher.com/jp/ja/home/technical-resources/mediaformulation.165.html

\begin{tabular}{llll} 
Components & $\begin{array}{l}\text { Molecular } \\
\text { weight }\end{array}$ & $\begin{array}{l}\text { Concentration } \\
(\mathrm{mg} / \mathrm{L})\end{array}$ & $\mathrm{mM}$ \\
Glycine & 75.0 & 750.0 & 10.0 \\
L-Alanine & 89.0 & 890.0 & 10.0 \\
L-Asparagine & 132.0 & 1320.0 & 10.0 \\
L-Aspartic acid & 133.0 & 1330.0 & 10.0 \\
L-glutamic acid & 147.0 & 1470.0 & 10.0 \\
L-Proline & 115.0 & 1150.0 & 10.0 \\
L-Serine & 105.0 & 1050.0 & 10.0 \\
\hline
\end{tabular}

Reduced representation bisulfite sequencing protocol

Referring the methods developed by Hongcang $\mathrm{Gu}$ and Patrick Boyle et al. [6, 7], we proceeded with the following protocol. Prior to thawing the blastocysts, DNA was rapidly collected using the QIAamp DNA Micro Kit (QIAGEN, Japan). The extracted genomic DNA was cleaved using restriction enzyme MSPI (C $\downarrow$ CGG) (Thermo Scientific, Japan). Upon performing gap filling with Klenow fragments (Thermo Scientific), A-tailing was performed. Thereafter, ligation was performed using L4 ligase (Thermo Scientific) and bisulfite conversion was performed using a bisulfite conversion kit (Methylode ${ }^{\mathrm{Tu}}$, Invitrogen). After purification, library amplification was performed by polymerase chain reaction (PCR) using a thermal cycler (Veriti ${ }^{\mathrm{m}}$, Applied Biosystems, Japan). The cycle conditions were set as $98^{\circ} \mathrm{C}$ for $45 \mathrm{~s}$, followed by $98^{\circ} \mathrm{C}$ for $15 \mathrm{~s}, 60^{\circ} \mathrm{C}$ for $30 \mathrm{~s}$, and $72^{\circ} \mathrm{C}$ for $30 \mathrm{~s}$ for total 18 cycles. To determine whether good amplification had been obtained, we verified that the 
target peak is obtained in electrophoresis using a 2100 Bioanalyzer (Agilent).

\section{Data collection}

Sequencing was conducted using the Hi-Seq ${ }^{\mathrm{Tm}} 1500$ (Illumina), and the FASTQ file was mapped using a program called the OKUMURA Pipeline NS0048 (National Center for Child Health and Development, Japan) which combines the following mapping programs: Bismark (www.bioinformatics.babraham.ac.uk/projects/bismark/), Bowtie (http://bowtie-bio.sourceforge.net/index.shtml), and Trim Galore (www.bioinformatics.babraham.ac.uk/ projects/trim_galore). Data such as the BAM text files were read and analyzed using the analysis software $\mathrm{R}$ (https://www.r-project.org/). In addition, the coverage and consistency of each sample in the data was visually verified using an IGV viewer (http://software.broadinstitute.org/software/igv/). Imprinted genes exhibiting major changes in methylation were extracted. All experiments on this study have been approved by the National Center for Child Health and Development. All experiments were performed in accordance with relevant guidelines and regulations.

\section{Statistical analysis}

Data were analyzed using the analysis software R. For methylation analysis, differentially methylated region (DMR) annotation and graph depiction, we used the MethylKit [8], edmr [9] and ggplot 2 of the R Package Software. For annotation, we used the Linux software Homer (http://homer.salk.edu/homer/). The terms hypermethylation and hypomethylation used in this report are defined as a change in methylation by $25 \%$ compared with blastocysts cultured in the KSOM culture medium alone [10].

\section{Results}

We used 26 female mice and collected a total of 469 ova. Ovulation was observed in 25 mice, and the mean number of ova per ovulation was 18.8. A total of 364 ova reached the blastocyst stage. With NoAA, 99 out of 131 ova reached the blastocyst stage, indicating a development rate of $75.6 \%$. Similarly, with EAAs, 81 out of 107 ova reached the blastocyst stage, indicating a development rate of $75.7 \%$. With NEAAs, 99 out of 128 ova reached the blastocyst stage, indicating a development rate of $77.3 \%$. With AllAA, 86 out of 103 ova reached the blastocyst stage, indicating a development rate of $83.5 \%$. Although there was no major difference in the blastocyst development rate with respect to the presence or absence of either essential or NEAAs, the development rate was lower in the culture medium with either essential or NEAAs than in the culture medium containing both essential and NEAAs.
The amount of specimens collected from all groups amounted to the $10 \mathrm{ng}$ input of genomic DNA (gDNA) required for RRBS. DNA was extracted using QIAamp ${ }^{\circ}$ DNA Micro Kit (50) (QIAGEN) from collected blastocysts and was cryopreserved without delay at $-80^{\circ} \mathrm{C}$, after which we proceeded with the RRBS protocol.

Figure 1 presents the results of the sequencing (Illumina Hiseq1500). Overall, reading was centered on the transcription starting site (TSS). Furthermore, a large proportion of $\mathrm{CpG}$ were observed in the exon and promoter regions (Fig. 2).

Next, we divided and compared the aforementioned region according to $\mathrm{CpG}$ islands (CGI) and non-CGI (Fig. 3). The proportion of CGI was high in the promoter and 5'UTR regions, which was consistent with the readings around the TSS.

Next, we compared each culture medium. The proportion of CpG sites with hypermethylation, hypomethylation and no change in each region is outlined in Fig. 4ad. With all culture media, demethylation was observed in approximately $100 \%$ of the promoter and 5 'UTR regions as a result of reprogramming. The overall distribution of methylation revealed hypermethylation in the intergenic and intron regions, with hypomethylation in TSSs, indicating that the results of the present study were consistent with those reported in previous studies [11]. Loci that maintained hypermethylation as a result of escaping demethylation through reprogramming was more abundant in NEAA compared with EAAs. Fisher's exact test was performed to compare hypermethylation in exons and promoter regions of EAAs (14937 loci), hypermethylation in in other loci (580415 loci), hypermethylation in exons and promoter regions by NEAAs (20619 loci), and hypermethylation in other loci (574733 loci). A significant difference was noted with a $p$ value of $2.2 \mathrm{e}-16(<0.05)$.

Next, using NoAAs as the control, we compared CpG with increases or decreases of over $25 \%$ for each culture medium (Fig. 5a-c). With NEAAs, most regions shifted to hypermethylation.

Fisher's exact test was performed to compare hypermethylation in exons and promoter regions with that in other regions between EAAs and NEAAs, and a significant difference was observed ( $p=2.2 \mathrm{e}-16)$. Similar comparison was made between AllAAs and NEAAs, which also showed a significant difference $(p=2.2 \mathrm{e}-16)$.

In the 5'UTR and promoter regions, the changes to hypermethylation and hypomethylation were extremely small for all culture media. Further, we analyzed imprinted genes in which methylation was observed to be considerably changed (file. 1.2). A total of 149 types of imprinted genes recorded on the Gene imprint (www. geneimprint.com/site/home) were extracted. As a result, we found this mapped to CGI at a total of 3304 sites of 


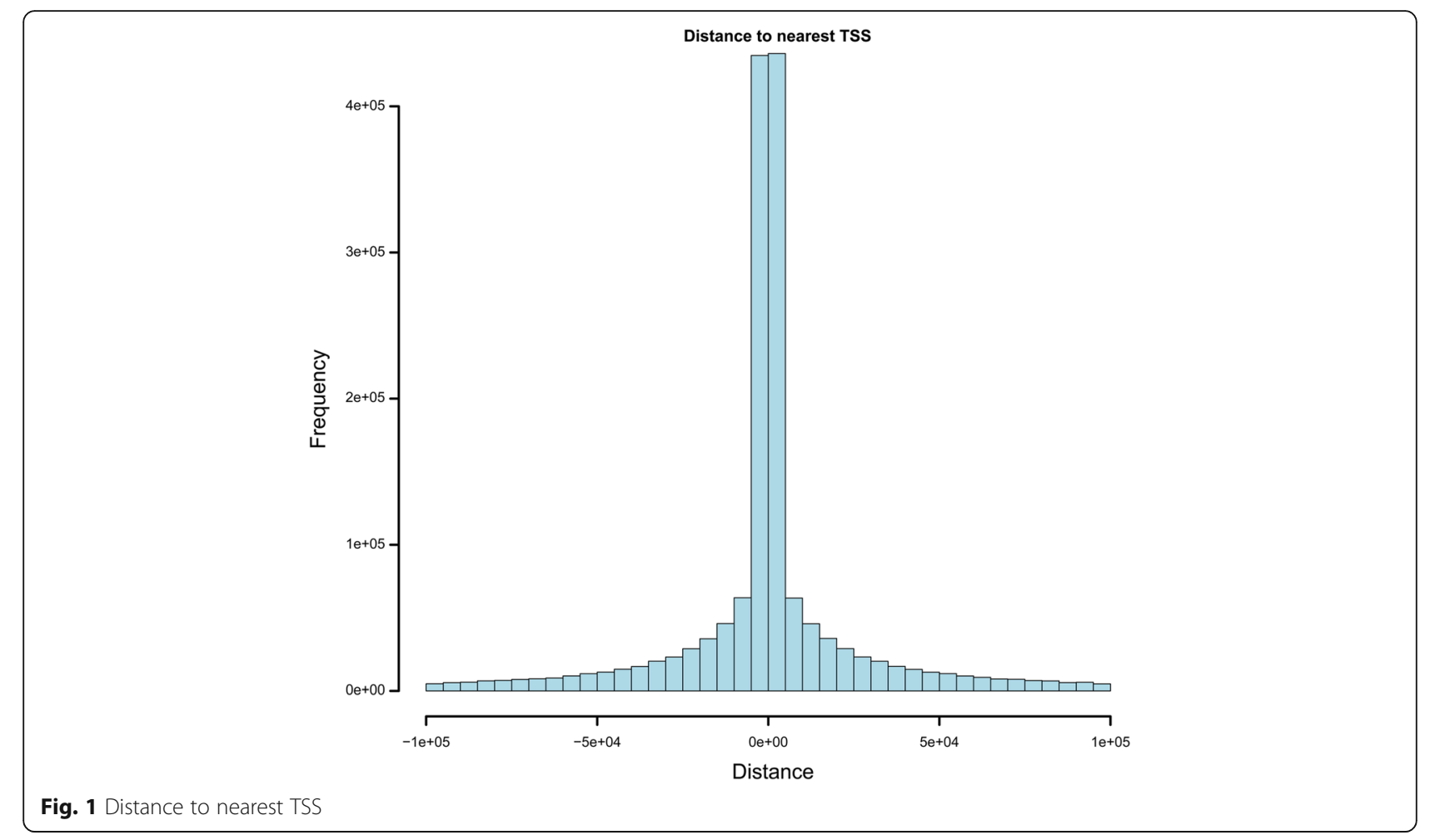

paternal genes and 2777 sites of maternal genes, for an overall total of 6081 sites.

Among which, using NoAAs as a control, we extracted sites showing changes of over $25 \%$ for all culture media, limited to the promoter and exon regions.

As a result, we extracted 532 sites on the paternal gene and 295 sites on the maternal gene. Genes extracted from the paternal gene included Zdbf2, Sfmbt2, Mcts2, Nnat, Nespas, Magi2, Peg10, Mest, Nap1l5, Peg3, Snrpn, Snurf, Magel2, Mkrn3, Peg12, Gab1, Mir184, Rasgrf1, Plagl1, Zrsr1, Dlk13, Peg13, and Jpx. On the maternal gene, we extracted Gatm, Ppp1r9a, Klf14, Zim1, Peg3os,

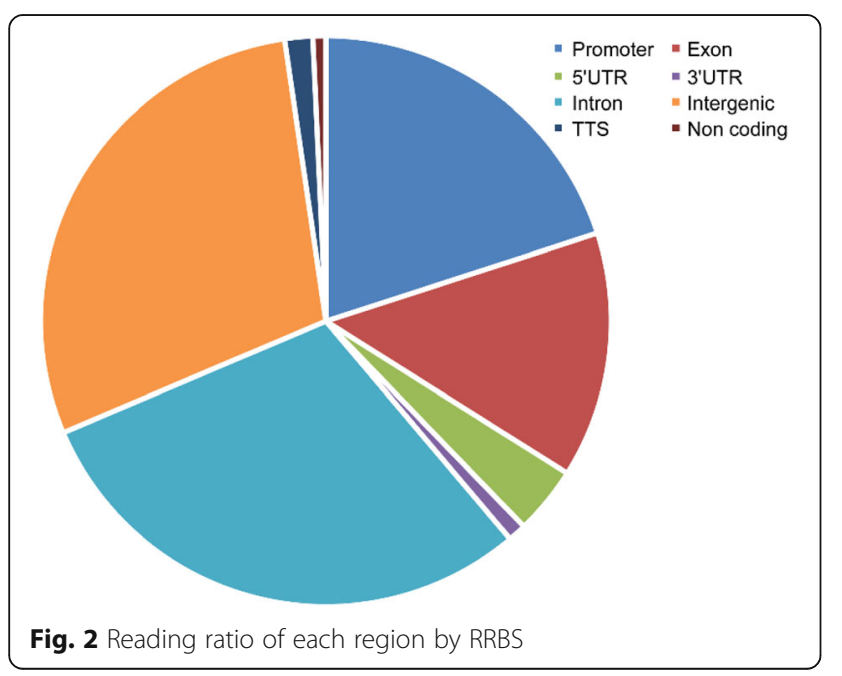

Zim3, Ampd3, H19, Th, Cdkn1c, Slc22a18, Nap1l4, Ano1, Mir431, Mir127, Kcnk9, Slc22a2, and Igf2r.

As a gene that showed distinct change, Mcts 2 showed hypermethylation only with NEAAs and hypomethylation in the other culture media. Nnat showed particularly high hypomethylation. On Nespas, hypomethylation was observed in all culture media, and Nap114 showed hypermethylation only with AllAAs (in other culture media, the methylation rate was 0\%). H19 exhibited a marked decrease in methylation with NEAAs.

\section{Discussion}

RRBS used in the current study has advantages such as the ability of conducting focused mapping on CpG-rich exon or promoter regions at a single base level, which is suitable for comprehensive analysis. Theoretically, analysis can be performed with as little as 1-10 ng of specimen; however, for other methods, gDNA of minimum volumes in $\mu$ grams is needed till date. Thus, to analyze methylation in early embryos, a large volume of specimens was needed. Therefore, RRBS is suitable for the analysis of methylation in early embryos with small amounts of gDNA [6,7].

A limitation is that RRBS is a semi-comprehensive analysis rather than a comprehensive analysis of methylation such as post-bisulfite adaptor-tagging. However, the results of the present experiment focusing on CpGrich sites are consistent with those of the comprehensive analyses. 


\section{Promoter Exon 4 \\ - CGI $=$ Non CGI \\ - $\mathrm{CGI}$ - Non CGI \\ 5'UTR

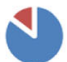 \\ - $\mathrm{CGI}$ - Non CGI \\ - $\mathrm{CGI}$ - Non CGI}

\section{Intron}
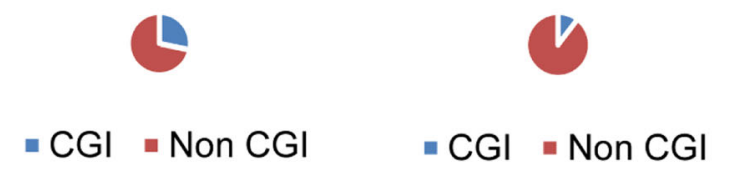

TTS

Whole region

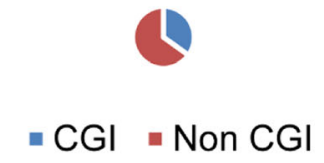

Fig. 3 Ratio of each region according to CpG islands (CGI) and non-CGl

A certain blastocyst sample volume is required for the RRBS protocol that is appropriate for analysis. If a large proportion of the blastocysts are in a methylated state that is largely deviated from others, this may affect the mean value obtained from analysis. Various reports indicate that the conditions of the culture medium affect the epigenetics of pre-implanted embryos, as observed in large offspring syndrome and Beckwith-Wiedemann syndrome $[12,13]$. Furthermore, depending on the amino acids and electrolyte composition of the culture medium, the blastocyst development rate remarkably changes $[14,15]$. In embryos that cease to grow or die, there are underlying problems of aneuploidy, metabolic requirements and ammonium production [16]; however, it is likely that the difference in the in vitro environment induced epigenetic changes, which consequently may have resulted in embryonic death [17].

In the history of culture media, Harrison successfully developed a medium in 1907. Since then, progress has been made with various approaches and improvements. In the 1950s, Whitten was the first to successfully culture eight-cell mouse embryos to the blastocyst stage [18]. Thereafter, the basic approaches to the most suitable culture medium have been the "let the embryo choose" principle and the "back to nature" principle [19]. The KSOM medium is a common culture medium based on the "let the embryo choose" principle. From the perspective of energy requirements switching from pyruvic acid in the early stage to glucose in the late stage of pre-implanted embryonic development [20], the KSOM medium contains well-balanced nutrients and the necessary buffer constituents.

When following the "back to nature" principle, another reason for selecting KSOM is that the in vivo environment changes with embryonic growth. It is believed that the medium should be close to the in vivo environment; thus, the concept of sequential media has gained popularity. However, continual changes in the culture medium increases stress on the embryo, and because the growth factors secreted by the embryo becomes diluted in the new medium, there are advantages and disadvantages to both a single medium and sequential media, and the question remains controversial [21]. In this study, we compared changes in methylation caused by the addition of amino acids and used KSOM as the basic medium.

It has been reported that amino acids show minimal impact in terms of blastocyst formation. However, with increasing concentration of amino acids, the probability of hatching increases, the number of inner cell masses increases and the formation of the extracellular matrix becomes closer to normal [16, 22]. Alternatively, it has been reported that EAAs reduce the blastocyst maturation and development rates, to which NEAAs show a compensatory effect. It has been reported that even with administration of NEAAs alone, good blastocyst outcomes are achieved [16, 23]. Conversely, Whitten and Conaghan developed embryos till the blastocyst stage in human and mice culture media without amino acids, and they reported that the 20 essential and NEAAs were not absolutely essential for growth [24]. Many other reports can also be found that question the most suitable constituents of culture media [25]. Ammonium is a product of amino acid metabolism; however, it is reported to be harmful for pre-implanted embryos [16]. The use of multiple culture media simulating the in vivo environment as much as possible has been found to improve embryo development [26]. As per the "let the embryo choose" principle, the most suitable medium for the embryo is experimentally determined [19], and as per the "back to nature" principle [27], the culture medium closely reproduces the in vivo environment. 

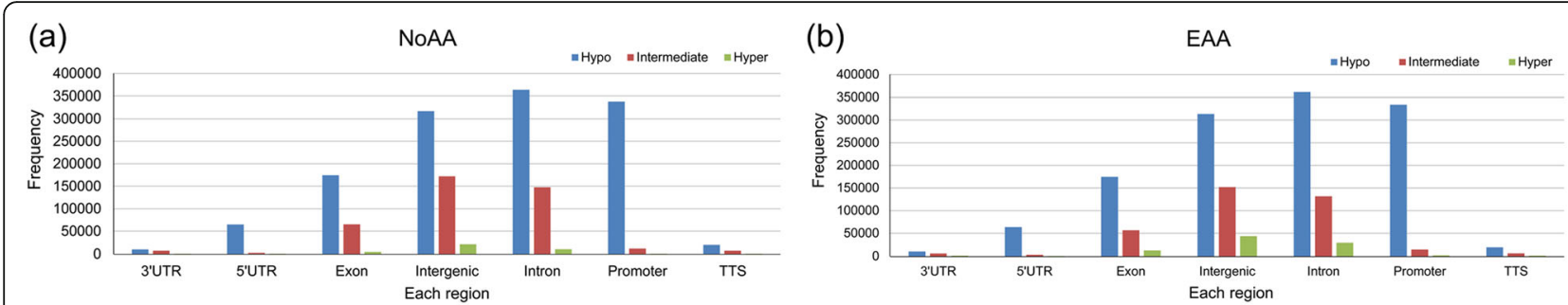

(c)

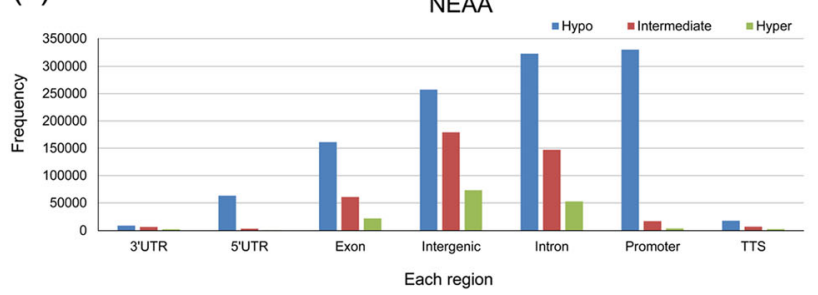

(d)

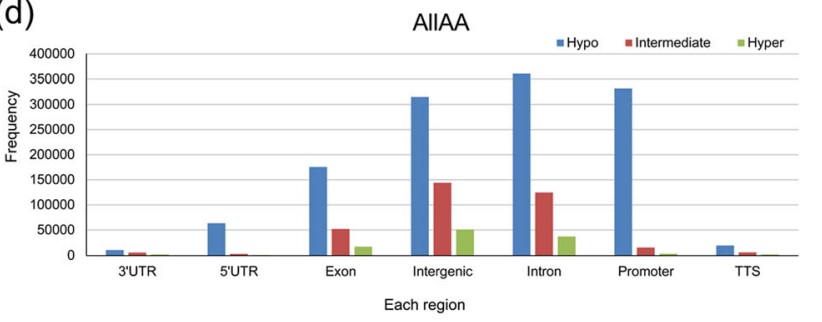

Fig. 4 Number of CpGs in the hypermethylation group, hypomethylation group, and intermediate methylation group shown per region. (> 75\%: hypermethylation; < 25\%: hypomethylation; other: intermediate group). a NoAA; b EAA; c NEAA; d AllAA; CpG: 5'-C-phosphate-G-3

The question as to which of these is most suitable for the embryo remains controversial [28].

The reports mentioned above describe results limited to specific gene loci, imprinted genes and DMR; thus, it is possible that methylation is disrupted at other genes that were not analyzed. Amino acids may exert subtle effects (not to a degree of exerting lethal genome-wide changes). To elucidate this, we added amino acids with different constituents to the KSOM culture. Although there was no major difference in the blastocyst development rate according to the presence or absence of added essential and NEAAs, the development rate was lower in culture media containing both essential and NEAAs. This suggested that both types of amino acids may be important for the initial stage of embryonic development.
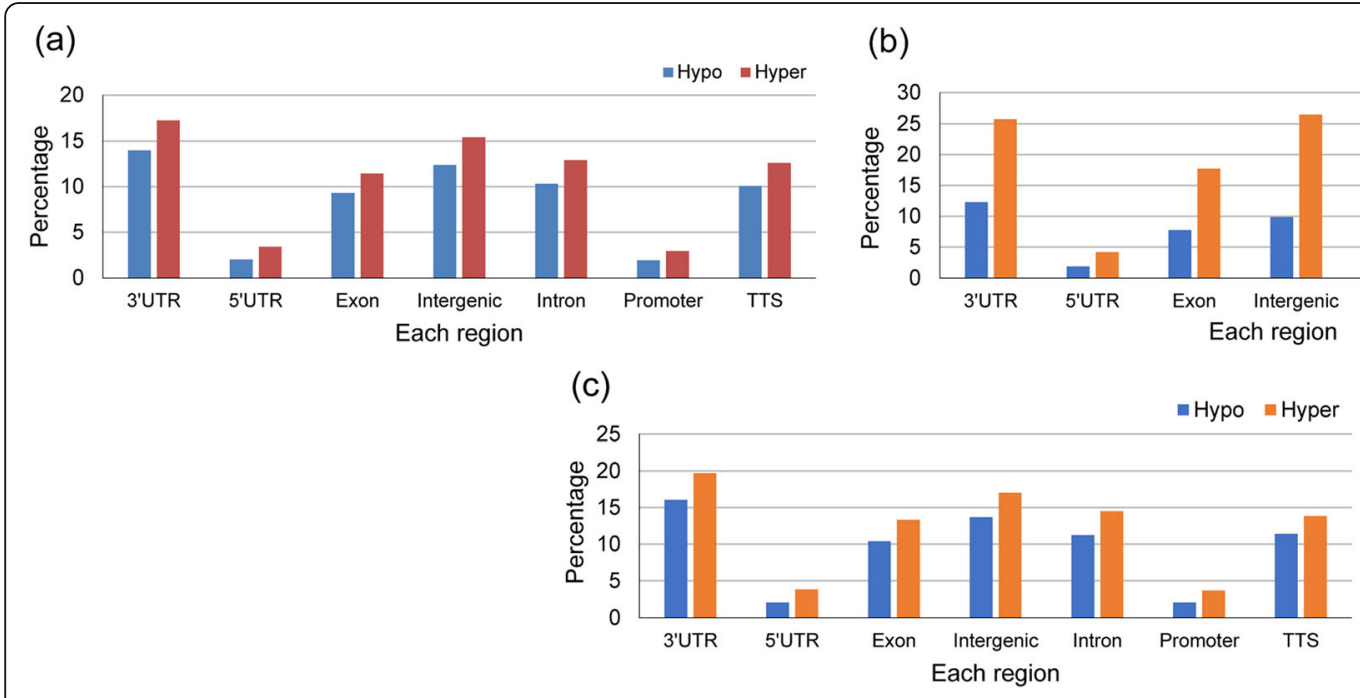

Fig. 5 Percentage of CpGs in the elevated methylation group and depressed methylation group compared with NoAA group is shown per region. (> 25\% elevated: hypermethylation group; > 25\% reduced: hypomethylation group). a EAA; b NEAA; c AllAA; CpG: 5'-C-phosphate-G-3

The current study is the first report that comprehensively analyzed changes in methylation according to different conditions of culture media in blastocysts.

In this study, we found that (1) uniform mapping was performed by RRBS; (2) there were many regions that maintained hypermethylation, particularly with NEAAs; (3) there was little overall effect of demethylation from reprogramming in the 5'UTR and promoter regions; and (4) specific changes were observed in imprinted genes, such as Nnat and Nespas.

Overall, results of our analysis revealed that CpG mapping was uniformly performed in all culture media, and there was no difference according to mapping between each culture medium, which shows that good data were obtained. This was also supported by the fact that CGI

(b)

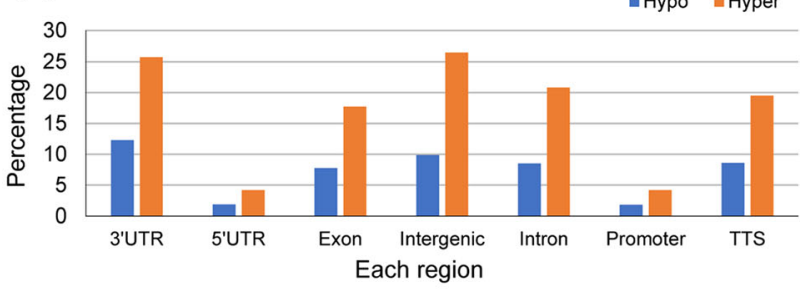


accumulation was mostly in CpG-rich promoter and exon regions (Figs. 1 and 2).

When focusing on each culture medium, as shown in Fig. 4, many sites were found that maintained methylation in medium with NEAAs compared with other culture media. This suggested that when compared with EAAs, NEAAs protected genes from demethylation caused by reprogramming.

In terms of protecting methylation disturbance, Fig. 5 shows that there was little change in methylation in the promoter and 5'UTR regions. This could suggest the presence of a mechanism to protect changes in methylation in regions that were important for translation from external factors (in the present study, to prevent stimulation, i.e., changes in amino acids).

Focusing on individual genes, we observed methylation changes in genes that have been reported to be defective in human imprinting disorders, such as the imprinted genes: Nnat, Nespas, Snrpn, Snurf, H19, and Mest.

As noted by Adam et al., changes in methylation of CpG in pre-implantation embryos are strongly affected by the culture medium constituents [29], and this also affects RNA expression such as in metabolism-related genes [23]. In the current experiment, we also found methylation changes in Acaca and Slc3a1 genes associated with metabolism.

In particular, methylation in the promoter region of Slc3a1 increased to hypermethylation only with NEAAs. We observed methylation disturbances in many of the same genes that have been previously reported to be prone to methylation disturbance owing to the external environment. In the present study, it was suggested that the addition of NEAAs induced a mechanism by which methylation was particularly preserved from external stimuli.

\section{Conclusion}

In recent years, it has become possible to comprehensively and efficiently examine methylation in specimens, and in the current study, we were able to extensively examine the effects of methylation. When comparing the current experiment with previous reports and comprehensively comparing methylation in human and mouse pre-implantation embryos, comparable results were achieved, which suggested that the changes observed in the current study could provide similar results in humans [30].

In conclusion, regarding how the changes in methylation observed in the current experiment are associated with gene expression, a report describing gene expression analysis is anticipated.

\section{Abbreviations}

AllAA: EAAs + NEAAs; ART: Assisted reproductive technology; CGI: CpG islands; CpG: 5'-C-phosphate-G-3'; DMR: Differentially methylated region; EAAs: Essential amino acids; gDNA: Genomic DNA; ICSI: Intracytoplasmic sperm injection; IVF: In vitro fertilization; NEAAs: Non-essential amino acids; RRBS: Reduced representation bisulfite sequencing; TSS: Transcription starting site

\section{Acknowledgements \\ Not applicable}

\section{Authors' contributions}

$\mathrm{KN}$ prepared the methodology. KN, KH, and HM supervised the study. MA put together the resources. KO handled the software. $\mathrm{KH}$ conceptualized the study and oversaw the project administration. All authors have read and approved the manuscript.

\section{Funding}

This work did not receive funding from any organization or institution. Experiment was conducted by mouse, approved on committee of National Center for Child Health and Development in Japan Approval number is 2010-002

\section{Availability of data and materials}

No changes required

Ethics approval and consent to participate

2010/February /1st IRB in National Center for Child Health and Development, Number 2010-002-C09

Consent for publication

Not applicable

\section{Competing interests}

The authors declare that they have no competing interests.

Received: 23 July 2019 Accepted: 21 November 2019

Received: 23 July 2019 Accepted: 21 Novem

\section{References}

1. Edwards RG (2001) The bumpy road to human in vitro fertilization. Nat Med. 7:1091-1094

2. Grafodatskaya D, Cytrynbaum C, Weksberg R (2013) The health risks of ART. EMBO reports. 14:129-135

3. Choufani S, Shuman C, Weksberg R (2010) Beckwith-Wiedemann syndrome. Am J Med Genet. 154C:343-354

4. Bowdin S, Allen C, Kirby G, Brueton L, Afnan M, Barratt C et al (2007) A survey of assisted reproductive technology births and imprinting disorders. Hum Reprod. 22:3237-3240

5. Hardarson T, Van Landuyt $L$, Jones G (2012) The blastocyst. Hum Reprod. 27: 72-91

6. Gu H, Smith ZD, Bock C, Boyle P, Gnirke A, Meissner A (2011) Preparation of reduced representation bisulfite sequencing libraries for genome-scale DNA methylation profiling. Nat Protoc. 6:468-481

7. Boyle P, Clement K, Gu H, Smith ZD, Ziller M, Fostel JL et al (2012) Gel-free multiplexed reduced representation bisulfite sequencing for large-scale DNA methylation profiling. Genome Biol. 13:92

8. Akalin A, Kormaksson M, Li S, Garrett-Bakelman FE, Figueroa ME, Melnick A, et al. methylKit: a comprehensive R package for the analysis of genomewide DNA methylation profiles. Genome Biol. 2012;13: R87.

9. Li S, Garrett-Bakelman FE, Akalin A, Zumbo P, Levine R, To BL et al (2013) An optimized algorithm for detecting and annotating regional differential methylation. BMC Bioinformatics. 14:S10

10. Market-Velker BA, Zhang L, Magri LS, Bonvissuto AC, Mann MR (2010) Dual effects of superovulation: loss of maternal and paternal imprinted methylation in a dose-dependent manner. Hum Mol Genet. 19:36-51

11. Guo H, Guo H, Zhu P, Yan L, Li R, Hu B, Lian Y et al (2014) The DNA methylation landscape of human early embryos. Nature. 511:606-610

12. Azzi S, Steunou V, Rousseau A, Rossignol S, Thibaud N, Danton F et al (2011) Allele-specific methylated multiplex real-time quantitative PCR (ASMM RTQ$P(R)$, a powerful method for diagnosing loss of imprinting of the 11p15 region in Russell Silver and Beckwith Wiedemann syndromes. Hum Mutat. 32:249-258 
13. Young LE, Fernandes K, McEvoy TG, Butterwith SC, Gutierrez CG, Carolan C et al (2001) Epigenetic change in IGF2R is associated with fetal overgrowth after sheep embryo culture. Nat Genet. 27:153-154

14. Erbach GT, Lawitts JA, Papaioannou VE, Biggers JD (1994) Differential growth of the mouse preimplantation embryo in chemically defined media. Biol Reprod. 50:1027-1033

15. Summers MC, Bhatnagar PR, Lawitts JA, Biggers JD (1995) Fertilization in vitro of mouse ova from inbred and outbred strains: complete preimplantation embryo development in glucose-supplemented KSOM. Biol Reprod 53:431-437

16. Gardner DK, Lane M (1993) Amino acids and ammonium regulate mouse embryo development in culture. Biol Reprod. 48:377-385

17. Rezk Y, Huff C, Rizk B (2004) Effect of glutamine on preimplantation mouse embryo development in vitro. Am J Obstet Gynecol. 190:1450-1454

18. Whitten WK, Biggers JD (1968) Complete development in vitro of the preimplantation stages of the mouse in a simple chemically defined medium. J Reprod Fertil. 17:399-401

19. Summers MC, Biggers JD (2003) Chemically defined media and the culture of mammalian preimplantation embryos: historical perspective and current issues. Hum Reprod Update. 9:557-582

20. Martin KL, Leese HJ (1999) Role of developmental factors in the switch from pyruvate to glucose as the major exogenous energy substrate in the preimplantation mouse embryo. Reprod Fertil Dev. 11:425-433

21. Lin S, Li M, Lian Y, Chen L, Liu P (2013) No effect of embryo culture media on birthweight and length of newborns. Hum Reprod. 28:1762-1767

22. Biggers JD, McGinnis LK, Raffin M (2000) Amino acids and preimplantation development of the mouse in protein-free potassium simplex optimized medium. Biol Reprod. 63:281-293

23. Park CH, Jeong YH, Jeong Yl, Kwon JW, Shin T, Hyun SH et al (2014) Amino acid supplementation affects imprinted gene transcription patterns in parthenogenetic porcine blastocysts. PloS one. 9:e106549

24. Conaghan J, Handyside AH, Winston RM, Leese HJ (1993) Effects of pyruvate and glucose on the development of human preimplantation embryos in vitro. J Reprod Fertil. 99:87-95

25. Mann MR, Lee SS, Doherty AS, Verona RI, Nolen LD, Schultz RM et al (2004) Selective loss of imprinting in the placenta following preimplantation development in culture. Development. 131:3727-3735

26. Gardner DK (1998) Development of serum-free media for the culture and transfer of human blastocysts. Hum Reprod. 13:218-225

27. Leese HJ (1998) Human embryo culture: back to nature. J Assist Reprod Genet. 15:466-468

28. Gardner DK, Lane M (1996) Alleviation of the '2-cell block' and development to the blastocyst of CF1 mouse embryos: role of amino acids, EDTA and physical parameters. Hum reprod. 11:2703-2712

29. Doherty AS, Mann MR, Tremblay KD, Bartolomei MS, Schultz RM (2000) Differential effects of culture on imprinted H19 expression in the preimplantation mouse embryo. Biol Reprod. 62:1526-1535

30. Smith ZD, Chan MM, Humm KC, Karnik R, Mekhoubad S, Regev A et al (2014) DNA methylation dynamics of the human preimplantation embryo. Nature. 511:611-615

\section{Publisher's Note}

Springer Nature remains neutral with regard to jurisdictional claims in published maps and institutional affiliations.

\section{Submit your manuscript to a SpringerOpen ${ }^{\circ}$ journal and benefit from:}

- Convenient online submission

- Rigorous peer review

- Open access: articles freely available online

- High visibility within the field

- Retaining the copyright to your article

Submit your next manuscript at $\boldsymbol{\nabla}$ springeropen.com 